\title{
AXILLARY ARTERY CANNULATION IN TYPE A AORTIC DISSECTION OPERATIONS
}

Eugenio Neri, MD

Massimo Massetti, MD

Gianni Capannini, MD

Enrico Carone, MD

Enrico Tucci, MD

Francesco Diciolla, MD

Edvin Prifti, MD

Carlo Sassi, MD
Background: Femoral arteries are the preferred site of peripheral cannulation for arterial inflow in type $A$ aortic dissection operations. The presence of aortoiliac aneurysms, severe peripheral occlusive disease, atherosclerosis of the femoral vessels, and distal extension of the aortic dissection may preclude their utilization. Axillary artery cannulation may represent a valid alternative in these circumstances. Methods: Between January 15, 1989, and August 20, 1998, in our institution, 22 of 152 operations $(\mathbf{1 4 . 4 \%})$ for acute type A aortic dissection were performed with the use of the axillary artery for the arterial inflow. Axillary artery cannulation was undertaken in the presence of femoral arteries bilaterally compromised by dissection in 12 patients $(54.5 \%)$, abdominal aorta and peripheral aneurysm in 5 patients $(22.7 \%)$, severe atherosclerosis of both femoral arteries in 3 patients $(\mathbf{1 3 . 6 \%})$, and aortoiliac occlusive disease in 2 patients $(\mathbf{9 . 1 \%})$. In all patients, distal anastomosis was performed with an open technique after deep hypothermic circulatory arrest. Retrograde cerebral perfusion was used in 9 patients (40.9\%). Results: Axillary artery cannulation was successful in all patients. The left axillary artery was cannulated in 20 patients $(90.9 \%)$, and the right axillary artery was cannulated in 2 patients $(9.1 \%)$. Axillary artery cannulation followed an attempt of femoral artery cannulation in 15 patients $(68.2 \%)$. All patients survived the operation, and no patient had a cerebrovascular accident. No axillary artery thrombosis, no brachial plexus injury, and no intraoperative malperfusion were recorded in this series. Two patients $(\mathbf{9 . 1 \%})$ died in the hospital of complications not related to axillary artery cannulation. Conclusions: In patients with type $A$ aortic dissection in whom femoral arteries are acutely or chronically diseased, axillary artery cannulation represents a safe and effective means of providing arterial inflow during cardiopulmonary bypass. (J Thorac Cardiovasc Surg 1999;118:324-9)
The he femoral artery is the most common site of cannulation for cardiopulmonary bypass in type A aortic dissection. However the presence of peripheral disease (such as aortoiliac aneurysms, distal extension of the aortic dissection into the femoral arteries, severe peripheral aortoiliac occlusive disease, and atherosclerosis of the femoral vessels) may preclude this option.

From the Istituto di Chirurgia Cardiovascolare Universitá agli Studi di Siena, Unitá Operativa di Chirurgia dell' Aorta Toracica, Siena, Italy.

Received for publication Nov 16, 1998; revisions requested Jan 19, 1999; revisions received March 2, 1999; accepted for publication April 5, 1999.

Address for reprints: Eugenio Neri, MD, Istituto di Chirurgia Cardiovascolare Universitá agli Studi di Siena, Policlinico le Scotte, Viale M. Bracci, 53100 Siena, Italy.

Copyright (C) 1999 by Mosby, Inc.

$0022-5223 / 99 \$ 8.00+0 \quad \mathbf{1 2 / 1 / 9 9 0 9 4}$
Axillary artery cannulation may represent a valid alternative in these circumstances, is easy to perform, and does not increase the risk of the procedure. This study reviews our experience with this technique.

Patients and methods

Patients. Between January 15, 1989, and August 20, 1998, in our institution, 22 of 152 operations (14.4\%) for acute type A aortic dissection were performed with the use of the axillary artery for the arterial inflow.

There were 16 men and 6 woman. The mean age of patients was 55.5 years (median, 62.5 years; range, 18-79 years). Seven patients (31.8\%) had clinical stigmata of Marfan's syndrome. Clinical characteristics of patients are presented in the Table I.

Indications for axillary cannulation. Axillary artery cannulation was undertaken in the presence of femoral arteries bilaterally compromised by dissection in 12 patients $(54.5 \%)$, 
Table I. Clinical characteristics of patients with acute dissection of the ascending aorta

\begin{tabular}{lc}
\hline Clinical background & \\
Median age (y) & 62.5 \\
Female (\%) & 27.2 \\
Hypertension (\%) & 81.8 \\
Smoking (\%) & 31.8 \\
Diabetes (\%) & 13.6 \\
Marfan syndrome (\%) & 31.8 \\
Claudication (\%) & 36.3 \\
Aneurysm (\%) & 22.7 \\
Angina (\%) & 0.0 \\
Renal insufficiency (\%) & 4.5 \\
Chronic obstructive pulmonary disease (\%) & 27.2 \\
Clinical condition & \\
Chest pain (\%) & 100 \\
Syncope (\%) & 0.0 \\
Hypotension (\%) & 36.3 \\
Aortic regurgitation (\%) & 72.7 \\
Pericardial effusion (\%) & \\
Cardiac tamponade (\%) & ${ }^{\dagger}$ \\
Cardiopulmonary resuscitation (\%) & 45.4 \\
Aortic rupture (\%) & 31.8 \\
Myocardial ischemia (\%) & 0.0 \\
Cerebral ischemia (\%) & 0.0 \\
Spinal ischemia (\%) & 0.0 \\
Abdominal visceral (\%) & 0.0 \\
Lower extremity ischemia (\%) & 0.0 \\
\hline
\end{tabular}

"Preoperative noninvasive diagnosis of pericardial effusion.

Pericardial effusion with hemodynamic significance.

abdominal aorta and peripheral aneurysm in 5 patients $(22.7 \%)$, severe atherosclerosis of both femoral arteries in 3 patients $(13.6 \%)$, and aortoiliac occlusive disease in 2 patients $(9.1 \%)$.

Axillary artery cannulation was successful in all patients in whom it was attempted. The left axillary artery was cannulated in 20 patients $(90.9 \%)$, and the right axillary artery was cannulated in 2 patients $(9.1 \%)$.

Axillary artery cannulation followed an attempt of femoral artery cannulation in 15 patients $(68.2 \%)$. In 3 cases severe atherosclerosis made femoral arteries unsuitable for cannulation; the other 12 patients experienced either distal dissection of the artery or inadequate back flow once opened.

Axillary artery cannulation was decided before the intervention, on the basis of the preoperative investigations, in 7 patients $(31.8 \%)$. In 4 patients, aortic atherosclerotic and aneurysmal disease were diagnosed before the hospital admission and signaled in the patients files; in 3 patients, abdominal aortic aneurysm, with parietal thrombus, was identified and evaluated before the operation by contrast computed tomography.

Preoperative investigations are listed in the Table II. No specific arteriographic visualization of the axillary artery was obtained before the operation.

All procedures were done as emergency procedures; the operative techniques used are summarized in the Table III; no
Table II. Diagnostic evaluation of acute dissection of the ascending aorta

\begin{tabular}{lcr}
\hline Diagnostic evaluation & $n$ & $\%$ \\
\hline Chest radiography & 22 & 100.0 \\
Transthoracic echocardiography & 22 & 100.0 \\
Transesophageal echocardiography & 14 & 63.6 \\
Contrast computed tomography scan & 16 & 72.7 \\
Magnetic resonance image scan & 2 & 9.1 \\
Angiography & 0 & 0.0 \\
Peripheral Doppler imaging scan & 7 & 31.8 \\
\hline
\end{tabular}

Table III. Aortic replacement operations

\begin{tabular}{lrr}
\hline Procedure & $n$ & $\%$ \\
\hline Distal & & \\
$\quad$ Ascending aorta replacement & 10 & 45.4 \\
Hemiarch replacement & 9 & 40.9 \\
Arch replacement & 3 & 13.6 \\
Proximal procedure & & \\
Aorto-aortic graft & 7 & 31.8 \\
Aorto-aortic graft and aortic valve repair & 4 & 18.2 \\
Aorto-aortic graft and aortic valve replacement & 1 & 4.5 \\
Aortic root homograft & 1 & 4.5 \\
Bentall procedure & 2 & 9.1 \\
Cabrol procedure & 5 & 22.7 \\
Coronary button (Carrel patch) & 2 & 9.1 \\
\hline
\end{tabular}

associated procedures were undertaken. Circulatory arrest was used in all patients; retrograde cerebral perfusion was used in 9 patients $(40.9 \%)$. The mean period of cooling for these patients was 32 minutes; the mean circulatory arrest time was 21 minutes (range, 18-52 minutes). The time for rewarming averaged 44 minutes. Mean cardiopulmonary bypass time was 168 minutes (range, 122-235 minutes).

Intraoperative flow assessment with the aid of transesophageal echocardiography was performed in 9 patients $(40.9 \%)$. No patients required repositioning of the arterial cannula for malperfusion.

Operative procedure. The left axillary artery was the preferential site for cannulation, and the choice of the right axillary artery was made only when the left axillary artery was pulseless or demonstrated attenuation or reduplication of the pulse.

All operations in this series were performed through a median sternotomy. In all cases, hypothermic circulatory arrest was a planned procedure to allow resection by means of an open technique.

In aortic dissection procedures, an arterial line with a side branch is routinely predisposed, which may be used for retrograde cerebral perfusion during circulatory arrest or for alternative arterial cannulation. Subclavian central venous lines, which may preclude axillary artery cannulation, are avoided. Arterial lines are routinely placed on both radial arteries. 


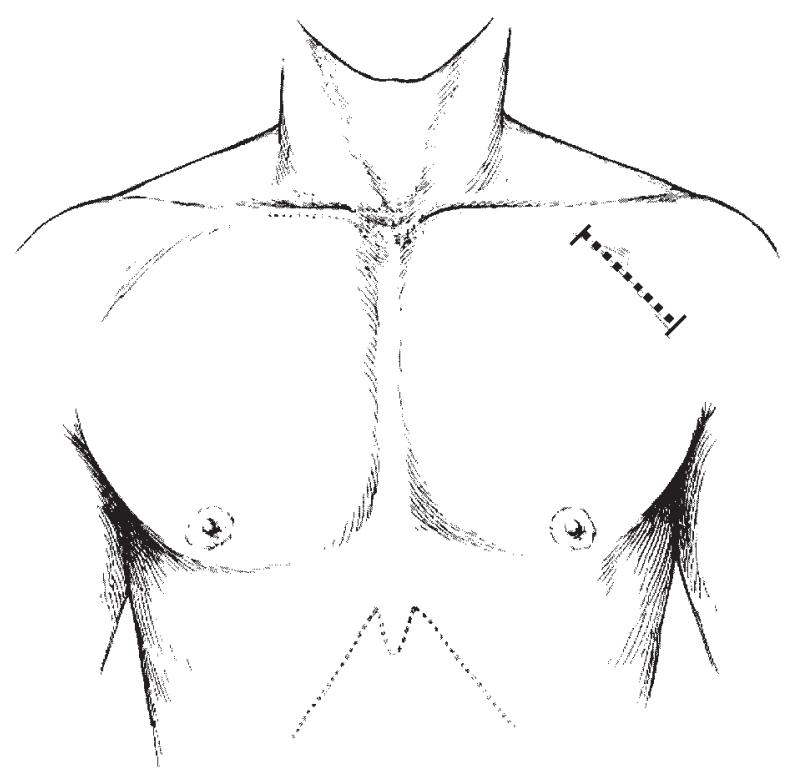

Fig 1. Site of incision of the deltopectoral groove for left axillary artery exposure.

The axillary artery exposure for cannulation is obtained through a 6- to $10-\mathrm{cm}$ incision over the top of the deltopectoral groove (Fig 1). The fibers of the pectoralis major muscle are split. The cephalic vein is encountered at this level, where it penetrates the fascia to join the axillary vein; it must be controlled and if necessary divided. The clavipectoral fascia is then incised, exposing the pectoralis minor muscle, which is divided or retracted laterally. Arterial branches of the thoracoacromial trunk will be encountered and if necessary divided to gain full access through the fatty tissue overlying the axillary vessels. In the dissection, the axillary vein is usually encountered first. The axillary artery lies superior and deep to the vein (Fig 2) and is readily palpated. If approached directly, following the thoracoacromial trunk, the artery is easily exposed and encircled by an umbilical tape. Gentle traction on the tape will allow the axillary artery to be drawn up into the wound, away from the cords of the brachial plexus, avoiding contact with this structure during the dissection. The thoracoacromial trunk is controlled by a tape and, after dissection over sufficient distance, does not need to be divided. Proximal and distal control of the axillary artery is gained, and the umbilical tape is passed through a tourniquet. After heparin is administered, femoral artery clamps are used proximal and distal to the cannulation site. A transversal incision is made, and the axillary artery is cannulated with either a 20 or 22 straight arterial cannula. The tourniquet is tightened, and the cannula is tied to the tourniquet. Flow is evaluated through the cannula by back bleeding, and if adequate, the cannula is connected to the arterial line and secured to the skin. The distal clamp is left in place until the end of cardiopulmonary bypass. Venous cannulation is performed with a 2-stage right atrial cannula. The superior vena cava is

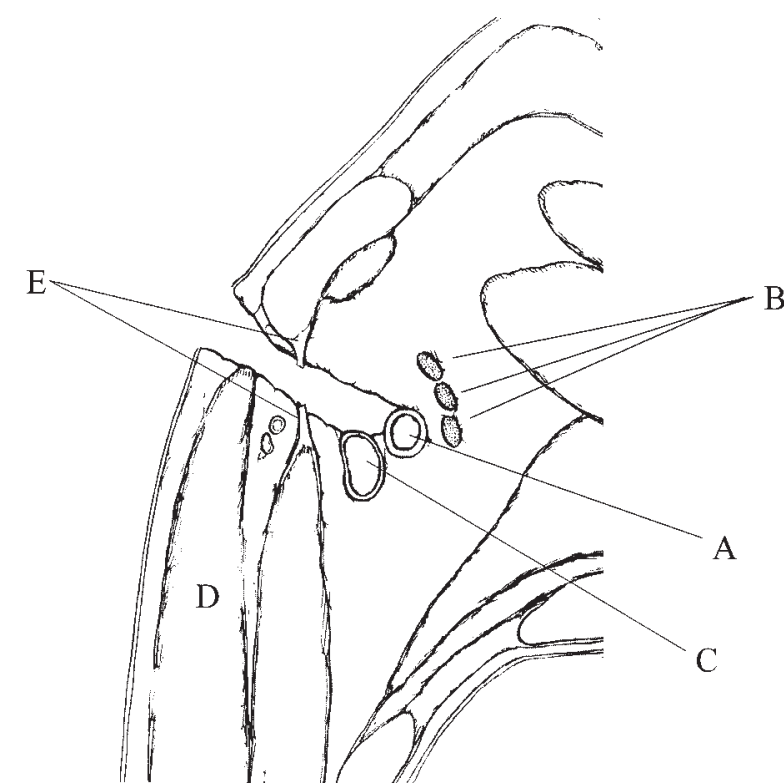

Fig 2. Diagram shows the anatomic relationship between the axillary artery $(A)$, brachial plexus cords $(B)$, and the axillary vein $(C)$. The arrow indicates the surgical access through the fibers of the pectoralis major muscle $(D)$ and the clavipectoral fascia $(E)$.

directly cannulated in patients who require retrograde cerebral perfusion during periods of circulatory arrest. Once cardiopulmonary bypass is established, systemic cooling is immediately initiated. Cardiopulmonary bypass flows, with cardiac indices of 2.0 to $2.5 \mathrm{~L} / \mathrm{min} / \mathrm{m}^{2}$, are readily obtained with axillary artery cannulation. The adequacy of arterial inflow is assessed clinically, and the following parameters are observed: pump flow resistance, a radial artery pressure drop, and the development of mydriasis. Transesophageal echocardiography is now routinely used for type A aortic dissection procedures to detect malperfusion of the supra-aortic trunks on initiation of cardiopulmonary bypass. Nasopharyngeal, esophageal, and bladder temperatures are monitored. An alpha-stat approach is used for acid-base management during hypothermia. Before hypothermic circulatory arrest, steroids are given routinely and the head is packed in ice to maintain cerebral hypothermia. Systemic cooling is considered to be adequate for hypothermic circulatory arrest when the nasopharyngeal temperature has reached $20^{\circ} \mathrm{C}$, and the bladder temperature is below $28^{\circ} \mathrm{C}$. At the desired temperature, the table is tilted down and the systemic arterial flow is then totally arrested. In patients in whom retrograde cerebral perfusion was performed, superior vena cava flow was adjusted to maintain a venous pressure of 25 to $30 \mathrm{~mm} \mathrm{Hg}$, which corresponded at a flow between 150 and $350 \mathrm{~mL} / \mathrm{min}$. Once proximal, the anastomosis is performed with an open technique and the aid of circulatory arrest, and the aorta is allowed to fill with blood with the pump turned manually by the perfusionist. The head is shaken by the anesthesiologist to 
dislodge any trapped air. At this point, the graft is clamped, and the systemic perfusion is restarted and rewarming is begun. Blood cardioplegia is administered intermittently directly through the coronary ostia. During rewarming, the aortic root portion of the procedure is completed. After the patient is weaned from the cardiopulmonary bypass, the arterial cannula is removed and the axillary artery is repaired.

\section{Results}

Deaths. Two deaths $(9.1 \%)$ occurred on postoperative days 8 and 16, respectively, as the result of the rupture of a known abdominal aorta aortic aneurysm and multiorgan system failure. No event was related to axillary artery cannulation.

Morbidity. All patients survived the operation, and no patient had a cerebrovascular accident. No axillary artery thrombosis, no brachial plexus injury, and no intraoperative malperfusion were recorded in this series.

Postoperative hemorrhage requiring reoperation occurred in 8 patients $(36.3 \%)$. Reoperation for late pericardial effusion was performed in 3 patients (13.6\%), respectively, on postoperative days 4,6 , and 9 .

Only 1 patient $(4.5 \%)$ needed prolonged postoperative inotropic support for low cardiac output. The same patient experienced sepsis and multiorgan system failure and died on postoperative day 16.

Postoperative atrial tachyarrhythmias were present in 3 patients (13.6\%). Six patients $(27.3 \%)$ underwent tracheotomy for prolonged ventilatory wean. One patient $(4.5 \%)$ with preoperative renal insufficiency required chronic hemodialysis treatment.

\section{Comment}

In operations for type A aortic dissection, the choice of the site of arterial inflow for cardiopulmonary bypass is heavily influenced by the idiopathic conditions. ${ }^{1-3}$ Femoral arterial cannulation, which is the standard method of cannulation for bypass initiation, has some disadvantages. The complex anatomy of the false lumen may acutely exclude the peripheral vessels, making femoral arteries unsuitable for the purpose. ${ }^{4}$ Retrograde perfusion through femoral cannulation may further elevate dissected intimal flaps ${ }^{5}$ and determine organ malperfusion, progressive arch vessel compromise, and neurologic injury. ${ }^{6}$ Furthermore severe atherosclerosis of femoral and iliac vessels may preclude femoral artery cannulation, and an increased risk of retrograde embolization of thrombus and atherosclerotic debris may derive from the presence of abdominal aortic or iliac aneurysmal disease. As a consequence of the great concern around these issues, alternative sites of arterial inflow have been described. ${ }^{4-11}$
Although we still use femoral artery cannulation in acute type A aortic dissection operations, we share the apprehension that such an avenue for arterial perfusion could itself cause or result in malperfusion or other serious complications. Therefore in all the cases in which the reliability of the femoral artery appears suboptimal, axillary artery cannulation is used. Particular attention is paid to the anatomy of the false lumen and its peripheral extension; the involvement of the abdominal aorta associated with reduced femoral pulses is considered a risk for peripheral cannulation. In recent years the use of axillary artery cannulation has become more common, and its routine application is a planned step.

It is evident that there is excellent flow through the axillary artery, associated with optimal cooling and rewarming. The advantage of relatively proximal cannulation and the consequent achievement of an antegrade flow throughout the complete descending aorta may prevent possible organ malperfusion as the result of the complex anatomic correlation between the perfusing and nonperfusing lumina. Furthermore, during deep hypothermic circulatory arrest, when the interior of the aortic arch is completely exposed, it is easy to check out the adequacy of the axillary artery by turning on the pump and observing the flow from the ostium of the subclavian artery (or innominate artery). With this maneuver we can rule out whether the axillary-subclavian axis is dependent on the false lumen and consequently foresee the need for recannulation of the prosthesis, in the eventuality that the false lumen is closed by the anastomosis.

Surgical exposure to the axillary artery is easy to perform, and if the operative field and the patient are routinely prepared for this procedure, it is as rapidly performed as femoral exposure.

Unlike the femoral vessels, the axillary artery benefits from rich collateral flow from the thyreocervical trunk to the suprascapular and transverse cervical arteries, avoiding the risk of upper extremity ischemia during total distal occlusion. ${ }^{12}$

In our practice we have rarely found heavily diseased axillary arteries, even in subjects with severe atherosclerosis who are undergoing axillobifemoral bypass. In this series of patients with type A aortic dissection, all the vessels were of excellent quality.

Involvement of an axillary artery by the dissection process is also rare. ${ }^{4}$ In this group of patients, we did not find any vessel with intimal flap formation.

Humeral pulse palpation allows for the detection of proximal involvement of the subclavian artery. A reduced pulse is likely due either to direct subclavian dissection or to ostial subclavian (innominate artery) 
involvement at the aortic level. In this circumstance cannulation of the axillary artery of that side is contraindicated.

This simple screening method is not possible for the femoral arteries, which are distant from the primary site of entrance, and their impairment may result from the complex anatomic interactions between the perfusing and nonperfusing lumen along the entire aorta. The left axillary artery is preferred over the right for cannulation. This choice precludes the option to selectively perfuse the brain, through the left carotid artery, during the period of deep hypothermic circulatory arrest. This is obtained by snaring or clamping the innominate artery. In the 2 patients of this series in whom the right axillary artery was cannulated, we did not use this technique, mainly because clamping or snaring the innominate artery may damage this structure. Furthermore antegrade perfusion is more cumbersome than retrograde cerebral perfusion, which in addition helps remove air and particulate emboli.

The other reason the left axillary route is preferred over the right is that the left subclavian artery has a separate and downstream origin from the carotid artery. This is a particularly important clue with regard to brain protection. At the beginning of perfusion, when the risk of embolization is higher, cardiac antegrade flow actually diverts microbubbles from the supra-aortic trunks and the particulate emboli released from the pump circuit and from the diseased vessels. Furthermore, in type A aortic dissection, the innominate trunk is often obstructed by expansion of the false lumen, and the right subclavian artery is obstructed more frequently than the left. ${ }^{4}$ In the same period covered by the present study, 152 patients with acute aortic dissection have received operative therapy in our institution. Femoral artery cannulation was undertaken in 130 patients. In this group, the mean age was 49.8 years (median, 61.2 years; range, 46-83 years). Preoperative stroke incidence was $4.6 \%$. Hospital death and neurologic injury rate were $19 \%$ and $11.5 \%$, respectively. Twenty-one patients $(16.1 \%)$ were treated with an aortic crossclamp technique, and an open technique repair was used in 109 patients. Retrograde cerebral perfusion was used in 32 patients, and selective antegrade perfusion in 21 patients. Documented intraoperative malperfusion was present in 18 patients $(13.8 \%), 14$ of which were repaired with an aortic crossclamp. Complete arch replacement was undertaken in 11 patients. None of the patients who received femoral artery cannulation had documented aortoiliac aneurysmal disease, and in all patients the femoral artery with the strongest pulse was chosen. Reperfusion back through the aortic graft was undertaken in 12 patients who received femoral artery cannulation.

These data summarize our results with femoral artery cannulation in type A acute aortic dissection operations over a 10-year period; the differences in the manner in which the procedures were performed and in the preoperative status are important. Comparison between the axillary artery group and the group who received femoral artery cannulation seems to indicate a superior outcome and a lower complication rate in favor of the axillary route. However, in the last 10 years, we have seen advances in the techniques and in the management of patients with type A acute aortic dissection. In this condition, the intrinsic difficulty is to discriminate whether the flow pattern, the pathophysiologic features, and the anatomic correlation between the perfusing and nonperfusing lumina expose to organ malperfusion. Introduction and intensive application of open technique of anastomosis, evolution, and use of cerebral perfusion techniques, amelioration of preoperative and postoperative management protocols, increased application of transesophageal echocardiography monitoring for malperfusion, and amelioration of perfusion devices may therefore account for a complication rate, in particular neurologic and malperfusion complications, not attributable only to the cannulation site.

\section{Conclusions}

In patients with type A aortic dissection in whom femoral arteries are acutely or chronically diseased, axillary artery cannulation represents a safe and effective means of providing arterial inflow during cardiopulmonary bypass.

In this condition the intrinsic difficulty is to discriminate the flow pattern, the pathophysiologic features, and the anatomic correlation between the perfusing and nonperfusing lumina exposed to the organ of malperfusion.

Only the prompt recognition of the problems and a compliant surgeon's repertoire may help in the management of these patients.

Although we strongly believe that no technique may shelter from these complications, the encouraging results with this technique appeal to us for the consideration of the axillary artery as the site of choice for cannulation in type A dissecting aneurysm.

\section{REFERENCES}

1. Baribeau YR, Westbrook BM, Charlesworth DC, Maloney CT. Arterial inflow via an axillary artery graft for the severely atheromatous aorta. Ann Thorac Surg 1998;66:33-7.

2. Bichell DP, Balaguer JM, Aranki SF, et al. Axilloaxillary cardiopulmonary bypass: a practical alternative to femorofemoral bypass. Ann Thorac Surg 1997;64:702-5. 
3. Sabik JF, Lytle BW, McCarthy PM, Cosgrove DM. Axillary artery: an alternative site of arterial cannulation for patients with extensive aortic and peripheral vascular disease. J Thorac Cardiovasc Surg 1995;109:885-90.

4. Borst HG. In: Borst HG, Heinemann MK, Stone CD, editors. Surgical treatment of aortic dissection. 1st ed. New York: Churchill Livingstone; 1996. p. 255-68.

5. Robicsek F, Guarino RL. Compression of the true lumen by retrograde perfusion during repair of aortic dissection. J Cardiovasc Surg (Torino) 1985;26:36-40.

6. Van Arsdell GS, David TE, Butany J. Autopsies in acute type A aortic dissection: surgical implications. Circulation 1998;98 (Suppl):II299-304.

7. Parr GVS, Manley NJ, Williams DR, et al. Obstruction of the true lumen during retrograde perfusion of type I aortic dissection: a simplified solution. Ann Thorac Surg 1980;30:495-8.

8. Neustein SM, Lansman SL, Quintana CS, Suriani R, Ergin MA,
Griepp RB. Transesophageal Doppler echocardiographic monitoring for malperfusion during aortic dissection repair. Ann Thorac Surg 1993;56:358-61.

9. Thomas CS Jr, Alford WC Jr, Burros GR, Frist RA, Stoney WS The effectiveness of surgical treatment of acute aortic dissection. Ann Thorac Surg 1978;26:42-9.

10. Najafi H, Veeragandham R. Thoracic aortic operations: management of maldistribution of arterial flow during cardiopulmonary bypass. Ann Thorac Surg 1997;64:565-7.

11. Yamada E, Matsumura M, Kimura S, et al. Usefulness of transesophageal echocardiography in detecting changes in flow dynamics responsible for malperfusion phenomena observed during surgery of aortic dissection. Am J Cardiol 1997;79:114952.

12. Gates JD, Bichell DP, Rizzo RJ, Couper GS, Donaldson MC. Tight ischemia complicating femoral vessel cannulation for cardiopulmonary bypass. Ann Thorac Surg 1996;61:730-3.

\section{Bound volumes available to subscribers}

Bound volumes of The Journal of Thoracic and Cardiovascular Surgery are available to subscribers (only) for the 1999 issues from the Publisher, at a cost of $\$ 134.00$ for domestic, $\$ 165.85$ for Canadian, and $\$ 155.00$ for international subscribers for Vol 117 (January-June) and Vol 118 (July-December). Shipping charges are included. Each bound volume contains a subject and author index and all advertising is removed. Copies are shipped within 60 days after publication of the last issue of the volume. The binding is durable buckram with the Journal name, volume number, and year stamped in gold on the spine. Payment must accompany all orders. Contact Mosby, Inc, Subscription Services, 11830 Westline Industrial Drive, St Louis, MO 63146-3318, USA; phone 800-453-4351 or 314-453-4351.

Subscriptions must be in force to qualify. Bound volumes are not available in place of a regular Journal subscription. 